\title{
Traduire une littérature mineure en langue majeure : quelques réflexions pour une traduction de Bord de Canal, roman martiniquais, en espagnol d'Espagne
}

\section{Translating a Minor Literature into a Major Language: Some Thoughts \\ for a Possible Translation into Castilian Spanish of Bord de Canal, a Martinican Novel}

RaQuel Gómez Pintado [raquel.gomezpintado@gmail.com]

Sorbonne Université, France

\section{RÉSUMÉ}

La mondialisation change notre façon de voir l'Autre, ce qui a une influence indéniable en traduction. Grâce à la des mouvements comme la mondialité, les auteurs postcoloniaux ont réussi à s'approprier la langue majeure en utilisant des stratégies hétérolingues, ce qui pose des défis en traduction. Le présent article analyse les enjeux d'une possible traduction en espagnol d'Espagne pour le roman Bord de Canal, de l'auteur francocréolophone Alfred Alexandre, se centrant sur certains des éléments hétérolingues présents dans le texte, à travers notamment des procédés proposés par Myriam Suchet.

\section{MOTS-CLÉS}

Bord de Canal ; hétérolinguisme ; mondialisation ; mondialité ; traduction postcoloniale

\begin{abstract}
Globalization has changed the way we see the Other. This change has an important impact on translation. Also, thanks to the process of mondialité, postcolonial authors have succeeded in appropriating the dominant language by using heterolingual strategies. This kind of strategy makes translation even more difficult. This article analyses some of the problems of a possible translation of the novel Bord de Canal, by the Frenchcreole-speaking writer Alfred Alexandre. It focuses on some heterolingual elements which appear in the text, using mainly the tools proposed by Myriam Suchet.
\end{abstract}

\section{KEYWORDS}

Bord de Canal; heterolinguism; globalization; mundanity; postcolonial translation

REÇU 2020-11-20; ACCEPTE 2021-01-15 


\section{Introduction}

Les réponses des auteurs francophones à la domination néocolonialiste favorisent la mise en valeur des langues et des cultures considérées jusqu’alors comme marginales, leur permettant d’affirmer une identité plurielle. À travers le lexique, la syntaxe et la morphologie, ces écrivains réinterprètent les règles du français, opérant un processus de reterritorialisation (Deleuze et Guattari, 1980) de la langue majeure ${ }^{1}$, pour y inscrire des pratiques linguistiques qui sont le reflet de leur société. Alfred Alexandre, écrivain martiniquais franco-créolophone, recourt à des stratégies hétérolingues dans son roman Bord de Canal ${ }^{2}$ (2004) pour montrer au lecteur une image «vraie» de la Martinique, loin des clichés tropicaux.

Les textes dans lesquels cohabitent plus d'une langue posent des problèmes singuliers en traduction. De plus, le travail de traduction se rend encore plus difficile lorsqu'il sagit de langues mineures. La traduction, abordée dans les travaux dérivant d'une position postcoloniale, a été appréhendée comme une pratique de domination, plus que comme un horizon d'échanges et de rencontres ${ }^{3}$. Néanmoins, certains auteurs considèrent que la mondialisation et l'affirmation des langues et des cultures « autres » invitent à lutter contre l'hégémonie occidentale du savoir, qui s'est aussi installée en traduction.

Nous avons entrepris d'analyser les enjeux de traduction de $B C$, d'Alfred Alexandre, à partir du postulat suivant : nous considérons que la traduction doit s'adapter au monde actuel, infiniment changeant et interconnecté, pour nous faire voir l'Autre. Ainsi, l’objectif de cet article sera de réfléchir, d'un point de vue postcolonial, autour des problèmes de traduction de certaines des stratégies hétérolingues présentes dans $B C$, et sur la façon dont la mondialisation (et plus précisément la mondialité) peut ouvrir la voie à une traduction respectueuse du Divers, de cette " différence consentie » (Glissant 1981 : 328), pour créer un texte-cible qui relèverait d'une traduction éthique (Berman 1992).

Afin de mener à bon terme cette analyse, nous nous centrerons d'abord sur l'influence de la mondialisation dans la traduction. Ensuite, nous analyserons les stratégies hétérolingues mises en œuvre par l'auteur afin de revendiquer son identité. Grutman (1997) parle d'hétérolinguisme pour faire référence à l'existence dans un texte littéraire d'idiomes étrangers, sous quelque forme que ce soit, ou de variétés (sociales, régionales ou chronologiques) de la langue principale. Finalement, nous nous interrogerons sur les possibles façons de traduire ces stratégies, nous basant principalement sur les outils présentés par Myriam Suchet (2009).

$1 \quad$ Nous utilisons dans cet article la distinction entre langue majeure et langue mineure à l'image des philosophes Gilles Deleuze et Félix Guattari. Dans leur œuvre Kafka, pour une littérature mineure (1975), ces philosophes ne parlent pas de langue, mais de littérature mineure, pour faire référence non à la littérature d'une langue mineure, mais à celle qu'une minorité fait dans une langue majeure. Une langue «mineure " serait donc celle qui fait un usage mineur d'une langue majeure. Les littératures dites mineures comme pourraient lêtre celles des Antilles françaises tendent à minorer une langue majeure comme le français en la confrontant à une langue mineure comme le créole ou les variétés régionales du français aux Antilles.

2 Désormais : $B C$

3 Voir par exemple les travaux de Venuti (1995), Robinson (1997) ou Suchet (2009). 


\section{Mondialisation et mondialité dans la traduction}

En Europe, la problématique culturelle en traduction s'est posée au fur et à mesure que la traduction s'est intéressée à un nombre plus ample de langues et que les théories ont évoluées. Pendant des siècles, les seules langues qui étaient considérées dignes dêtre traduites (en littérature) étaient les langues anciennes, qui occupaient le sommet de la hiérarchie linguistique. Ainsi, les problèmes culturels ne se posaient pas vraiment, jusqu’à ce la traduction accueillit de plus en plus de langues. L'un des premiers à sêtre sérieusement demandé la façon dont les éléments culturels devaient être traités lors de la traduction fut Eugene Nida, le célèbre traducteur de la Bible. Dans son article "Linguistics and Ethnology in Translation Problems », paru en 1945, il propose d'intervenir dans le texte pour adapter les références culturelles à la réalité du public-cible, en utilisant ce qu’il appelle les équivalences culturelles.

Après Nida, au fil du temps, les approches communicatives et socioculturelles de la traduction ont donné lieu à différentes techniques pour répondre à ce problème. D’aucuns ont choisi des stratégies telles que l'adaptation ou la domestication, qui effacent de la traduction les éléments susceptibles de créer une sensation détrangement lors de la lecture. Cette position a prévalu pendant des années, ce qui nous rappelle qu'en Occident au moins, les théories de la traduction, leur terminologie et leurs techniques ont été développées pour et par la traduction de langues majeures. Plus tard, cependant, d'autres traducteurs ont plutôt défendu le besoin de préserver la culture du textesource. Ces derniers utilisent des concepts développés, par exemple, dans la théorie fonctionnaliste de Nord, qui tient compte des différences culturelles des deux langues mises en relation ; dans l'approche variationnelle de Hewson et Martin, qui souligne le rôle du traducteur comme " opérateur culturel»; des concepts de l'école connue sous le nom de manipulation school, qui met en lumière les processus de manipulation qui ont lieu en traduction littéraire afin d’orienter les divers éléments culturels à la langue-cible (Hurtado Albir 2017). D’autres voix ont surgi à partir des années 1990, qui ont contribué au développement d’une approche plus idéologique concernant la culture et la traduction.

En effet, certains auteurs considèrent que la mondialisation constitue un moment propice aux échanges culturels. Les flux migratoires ont contribué au contact des civilisations, créant des nouveaux rapports entre les peuples et des nouvelles manières de se comprendre soi-même et de comprendre l'Autre. Ainsi, ces déplacements des populations ont mis en question l'idée d'identité comme une notion bien délimitée et pure. Cette situation résulte en une nouvelle façon de voir la propre culture et en la mise en question des rapports centre/marge depuis lesquels l'Occident a traditionnellement pensé le monde.

Le monde est en «babélisation » croissante, déclare Oustinoff (2011), et non seulement le monopole linguistique des anciennes époques nest plus envisageable, mais les langues occidentales perdent leur place dominante. Certaines études concernant l'influence de la mondialisation sur les rapports entre les langues ont été publiées, notamment dans la revue Hermes ( $\mathrm{n}^{\circ} 49$ et 56) et dans louvrage Traduction et mondialisation (2011). En ce sens, Raphaël Confiant (2016) affirme que la mondialisation permet aux langues minoritaires d'intégrer l'univers de la traduction. Cette deuxième mondialisation se différencie de la première, qui eut lieu à la fin du $X V^{e}$ siècle, en ce quelle est une revendication des cultures «à la marge » et non une imposition par la force d'une culture et d'une langue par l'Occident. 
Pour Édouard Glissant, la mondialisation c'est le côté négatif de ces rapports entre cutures : la monotonie, l'égalisation, le nivellement par le bas. Ainsi, afin que l'individu ne se perde pas dans ce processus d'échange, il propose la notion de mondialité, une poétique active permettant d'échanger avec les autres sans s'y diluer en ce processus. Selon l'auteur, la mondialité, c'est " penser et agir dans cet inextricable du monde, sans le réduire à nos propres pulsions ni intérêts, individuels ou collectifs, et surtout, à nos systèmes de pensée " (2005 : 22-23). Ainsi, la mondialisation serait le "revers névrotique» (2005: 138) de la mondialité.

Puisque l'Autre vit désormais parmi nous, Confiant affirme que l'Europe et les États-Unis se trouvent face à une « omniprésence [...] de l'Autre. Un Autre qu'ils ne peuvent plus, qu'ils ne semblent plus en mesure de digérer et de cannibaliser comme ils ont fait durant des siècles » (2016 : 240). Par le passé, en effet, l'Autre était l'inconnu absolu. Il se trouvait non seulement éloigné du point de vue culturel, mais souvent létait aussi physiquement, rendant plus facile la survivance des préjugés. Mais la mondialité a modifié les relations entre les différentes cultures, nous forçant à voir l'Autre. C'est cette sensibilisation à l'existence de l'Autre, qui finalement ose se nommer et ainsi rentrer dans la conscience du monde, qui permet de réfléchir autrement aux échanges culturels, à la relation avec l'Autre et donc aussi à la traduction. De nos jours, la revendication des marges traditionnelles permet le développement d'un modèle moins eurocentrée et, en conséquence, plus ouvert au Divers. Car, d'après Glissant, il y a un homme nouveau, qui vivait l'absolu mais qui est désormais appelé à vivre le relatif : "J'appelle relatif le Divers, la nécessité opaque de consentir à la différence de l’autre [...]. J’appelle absolu la recherche dramatique d'imposition d'une vérité à l'Autre» (1981:440).

En même temps que la mondialisation, et en étroite relation avec celle-ci, se sont développées les théories postcoloniales. Le terme postcolonial comprend toute la culture qui sest vue affectée par l'impérialisme, depuis lépoque de la colonisation et jusquà nos jours (Ashcroft, Griffiths et Tiffin 1989). Ces théories se centrent sur l'étude des mécanismes mis en place par l'appareil colonial et sur la façon dont les peuples dominés contestent l’oppression du centre. En tant qu’ancienne colonie de la France, il nous semble que tant la Martinique que les auteurs issus de ce territoire, dont Alexandre, peuvent être analysés sous cette optique.

L’un des instruments privilégiés de contestation des écrivains postcoloniaux c'est l'appropriation de la langue majeure. Le combat linguistique est fondamental dans ce type de sociétés, car la revendication d'une langue implique aussi la revendication du droit d'exister dans une culture et dans une forme de vie choisie (Calvet 2001). Ainsi, nous analyserons la façon dont Alfred Alexandre s’approprie le français dans son œuvre $B C$ pour revendiquer son identité.

\section{Hétérolinguisme et revendication identitaire dans Bord de Canal}

De nous jours, comme conséquence de l'histoire coloniale du territoire, deux langues cohabitent en Martinique : le français, langue officielle de par l'appartenance politique de lîle à la France, et le créole, dépourvu du statut de langue officielle mais parlé par l'ensemble de la population. D’après Georges Daniel Véronique (2000), le mot créole est attesté en français dès 1649, d’abord pour faire référence à des " baragouins », « jargons », « langages nègres » ou " patois nègres ». Ce type de 
langues, rappelle l'auteur, sont nées comme résultat de relations asymétriques entre les Européens et les populations Africaines importées dans les colonies.

Les conditions liées à la création du créole, que nous pourrions qualifier de "traumatiques ", font que, actuellement, il est rejeté par une partie de la population, notamment par la classe moyenne, qui voit dans le français une façon de se débarrasser du poids colonial et de se rapprocher des classes dominantes (Affergan 2006). Cette réalité fut dépeinte par Patrick Chamoiseau dans Chemin-d'école (1994), où l'auteur évoque l'interdiction de parler créole à l'école et le rapport problématique des élèves au français. Dans le cadre littéraire, le créole a été mis à l'écart pendant longtemps par les écrivains euxmêmes : s'il est vrai que les doudouistes introduisaient parfois des mots en créole, ceci était uniquement fait avec une intention exotisante. Il faut attendre le roman Malemort de Glissant (1975), ainsi que la naissance de la Créolité pour voir apparaître dans la littérature antillaise l'utilisation du créole avec une signification identitaire.

En effet, le choix qui se pose à l'écrivain martiniquais entre une seule langue, le créole ou le français, est, en réalité, une farce héritée de l'époque coloniale, qui associait une identité unique a une seule langue. Mais la mondialité contribue à effacer les barrières strictes entre langue et identité. Glissant (1981) avait déjà annoncé la fin des "monolinguismes impérialistes ", comme le démontre lui-même dans Malemort, mais aussi des auteurs tels que José María Arguedas ou les tenants de la Créolité. Dans son bel ouvrage Écrire en pays dominé (1997), Chamoiseau retrace le cheminement intérieur qui lui permit décrire en utilisant les deux langues qui sont les siennes. Suivant la trace de ces écrivains, Alfred Alexandre trouve la liberté d'utiliser dans son texte le français et le créole pour souligner son identité multiple.

Dans BC, Alexandre recourt à l'hétérolinguisme (Grutman 1997) pour affirmer son identité " autre » par rapport à celle du centre hexagonal. Les stratégies hétérolingues, qui soulignent plutôt la différence ( "hétéro- ») que la multiplicité ("pluri- ») de langues dans un récit, consistent à mettre en place au sein d'un même texte non seulement des langues différentes, mais aussi des variétés sociales, régionales ou chronologiques de la langue. Dans cet article, nous analyserons deux des stratégies hétérolingues qui contribuent à manifester la volonté de l'auteur de $B C$ de mettre en évidence son identité multiple. Il s'agit de l'utilisation du créole et de la mise en relief du délire verbal.

La langue principale d'écriture dans $B C$ est le français, mais il est parsemé de mots créoles s'inscrivant dans le texte avec une graphie française, à commencer par le titre de l’ouvre, qui enferme une allusion au quartier martiniquais Bò kannal, où se situe l'action. Ainsi, nous trouvons dans le récit des mots tels que vagabonne ou jobeuse, alors que leur graphie créole est vakabòn et djobeuse, mot formé sur le verbe créole djobé. Il existe aussi des mots qui apparaissent dans le récit avec leur écriture originelle, car celle-ci ne rentre pas en conflit avec l'orthographe française, comme c'est le cas de tafia ou ababa.

Ainsi, ces mots, qui ne présentent d'ailleurs aucun balisage, s'insèrent dans le récit, conférant au texte, au niveau des signifiants, l’aspect d'un récit rédigé dans un français presque hexagonale. Or, la signification de ces mots peut rester obscure pour un lecteur non créolophone. Alfred Alexandre réussit donc à donner à son texte une certaine opacité (Glissant 1981), condition qui nous semble essentielle pour le respect entre cultures car elle permet d'en conserver leur originalité. En outre, il se détourne de la langue majeure, le français, en y introduisant des mots qui lui sont a priori étrangers. 
Une autre manière de s'approprier et de détourner la langue française est la création lexicale, que ce soit à travers du français des Antilles ou d’expressions à base créole. Ainsi, par exemple, à partir du verbe créole déchouké : « déracinement, chasser quelqu'un du pouvoir » (Confiant, 2007 : 312), Alexandre crée l'expression se faire déchouquer. Concernant le français des Antilles, il utilise par exemple le mot major, employé en Martinique pour parler d'un "fier-à-bras de quartier » (2007 : 920) et dont la graphie créole est majò. Dans ces deux exemples, l’auteur réussit à s'approprier le français à travers le mélange de celui-ci avec le créole.

L'autre stratégie hétérolingue que nous analyserons consiste à l'insertion du délire verbal dans le récit. Nous considérons le délire verbal comme une stratégie hétérolingue car ces stratégies font aussi référence aux variantes sociales; il nous semble que nous pouvons considérer ce type de discours comme une variante sociale à l'intérieur de la société martiniquaise. Cette stratégie contribue à donner une voix aux marginaux, l'un des buts du courant postcolonial, ainsi quà la création d'une image « vraie » de la Martinique, qui ne se présente plus comme un paradis tropical éloigné de tous les problèmes, mais qui s'inscrit dans le monde et, plus encore, dans une situation de violence urbaine ${ }^{4}$ profonde comme conséquence du rôle du territoire pendant la colonisation. Ainsi, la langue des marges s’insère dans la langue européenne.

Comme Jules Michelet Mambi le rappelle, «les grands traumatismes qu'a connus l'homme noir dans l'histoire, traite négrière, colonisation, ont inéluctablement eu une influence non négligeable dans la destruction de son psychisme » (2013 : 25). Dans le roman, nous constatons la présence d'une violence structurelle «née d'un processus économique et politique plaçant l'individu dans des conditions de vie impossibles» (2013 : 72). Les héros du roman, réduits à vivoter dans de conditions misérables, en sont un excellent exemple. Ces violences subies ont une incidence sur la psyché des personnages, qui se manifeste à travers des hallucinations et des délires, ainsi que des névroses, qui « constituent un morcellement des affects et un bouleversement de la personnalité » (2013 : 88).

Pour refléter dans le texte ces délires, l'auteur se sert de plusieurs outils. D’abord, le narrateur utilise toujours le pronom personnel « on » au lieu de " nous ", pour montrer son appartenance à un groupe qui constitue son identité, comme par exemple : "on voulait pouvoir se regarder en face, on leur disait, ne pas avoir à écouter notre miroir nous lancer qu’on était rien que des mendiants » (Alexandre 2004 : 134). C'est aussi une manière de prêter sa voix aux marginaux et de leur permettre d’exprimer leur désarroi. Pour démontrer le détachement du narrateur de la réalité, le récit est parsemé de phrases entrecoupées et d’autres construites avec une syntaxe désordonnée, qui sont le résultat d'une parole de la sensation : «Et c'est une vie entière de frustration et de petites compromissions quà travers son chant sans parole Clara exprimait, une vie faite de violences contenues, de petits meurtres interdits et en cette lente maturation des aurores, pour elle, c'est la nuit entière du ressentiment qui pointait » (2004:63).

Les choix de ponctuation d'Alexandre, assez inhabituels, permettent de créer un discours continuel. Ainsi, la répétition incessante des conjonctions copulatives ou, par contre, leur absence, contribuent à rythmer le récit : « Non amour, non, ne viens pas, ça nous emportera, ce sera trop haut trop beau trop fort » $(2004$ : 156). En outre, l'utilisation des virgules et des points dans le texte ne répond pas souvent aux normes de ponctuation classiques, car elle représente la langue orale : 
«C’est des accidents qui nétaient pas rares. Et qui arrivaient bêtement. Un soir, un diable, jailli de l’ombre. Et c’en était fini de vous » 2004 : 75). Une autre manière de traduire les déséquilibres mentaux des personnages est l'utilisation des répétitions, que ce soit du sujet ou du complément, sous la forme pronom + nom, pour montrer l'obsession : " De temps à autre, il y avait des pauses dans la méfiance, des interstices comme il disait Pépi » (2004:45-46), ou encore "On comprenait, Clara, qu'elle ait flanché) (2004 : 74).

Finalement, la dernière stratégie qui contribue à la mise en relief du délire verbal est l'utilisation de l'humour. Certes, l'humour pourrait servir à montrer un imaginaire créole, comme le suggère Confiant (2016), mais Ashcroft et al. donnent une explication différente de sa fonction dans les sociétés post-esclavagistes :

Subject to a tragic alienation from both language and landscape, the transplanted Africans found that psychic survival depended on their facility for a kind of double entendre. They were forced to develop the skill of being able to say one thing in front of 'massa' and have it interpreted differently by their fellow slaves. This skill involved a radical subversion of the meanings of the master's tongue (1999 : 146).

L'humour servirait donc à jouer avec la langue pour se faire comprendre uniquement des membres de l'endogroupe. Ainsi, l'humour dans $B C$ prend la forme d'une ironie « à la créole ». À travers cette ironie, le sujet créole propose une réponse critique à une situation donnée, de façon à ce quelle ne soit perçue comme telle que par ceux qui partagent le même code que lui. Il s’agit donc d'une forme de Détour, que Glissant définit comme suit : « Le Détour n’est pas un refus systématique de voir. Non, ce n'est pas un mode de la cécité volontaire ni une pratique délibérée de fuite devant les réalités. Nous dirons plutôt qu'il résulte, comme coutume, d'un enchevêtrement de négativités assumées comme telles. [...] Le détour est le recours ultime d'une population dont la domination par un Autre est occultée» (Glissant $1981: 48$ ).

Affergan (2006), de son côté, voit dans l'utilisation de l'humour une stratégie d'évitement qui viserait à se cacher la réalité pour la rendre plus supportable. L’exemple suivant reflète bien l'utilisation de cet humour ironique dans le roman. Le héros explique les représailles auxquelles les clandestins devaient faire face s'ils n'arrivaient pas à payer leur loyer : «Oh ! pas des choses extravagantes : une raclée, un coup de lame, voire, un soir de lune faible, une ou deux balles encastrées dans les omoplates» (2004:18).

\section{Nos stratégies de traduction face aux stratégies hétérolingues de Bord de Canal}

En tant que traductrice de Bord de Canal, il nous semble que le traducteur doit avoir une connaissance profonde de la réalité martiniquaise pour comprendre la pertinence de la structure et des éléments linguistiques dans le texte et pouvoir les traduire correctement. Il est donc essentiel de récréer dans la langue-cible les structures syntaxiques de type asyndète si fréquentes dans l'œuvre et qui relèvent de la déconnexion du narrateur avec la réalité. De plus, nous pensons qu'il est nécessaire d'introduire dans le texte-cible des choix de ponctuation qui permettent de refléter un 
discours continuel, comme la répétition des conjonctions copulatives ou l'absence déléments de ponctuation. En outre, même si l'ordre des phrases peut sembler chaotique, nous trouvons fondamental de le conserver dans la mesure du possible afin de créer chez le lecteur-cible un étrangement similaire à celui ressenti par le lecteur-source. Rappelons qu'Alexandre a choisi cet ordre pour suggérer une parole de la sensation et les troubles mentaux du narrateur. En outre, il faudrait viser à conserver les fragments humoristiques et ironiques du texte, qui constituent une stratégie dévitement de la réalité.

Concernant le créole inséré dans le texte, il est, à notre avis, la stratégie hétérolingue qui peut poser le plus de problèmes au traducteur. Les approches concernant la traduction de textes dans lesquels plusieurs langues cohabitent sont divergentes. Certains théoriciens considèrent qu'il est impossible de traduire ce phénomène ; ils remplacent alors ces marques hétérolingues par des mots vulgaires, de remplissage... permettant de marquer le discours sans l'inscrire dans un territoire particulier (Hurtado Albir 2017). Cependant, le choix de rendre plus neutre le texte-cible contribue à effacer l'appartenance du texte-source à sa culture ou peut dériver dans une fausse représentation de la culture du texte (Anderson 2017). De plus, ce type de traduction gomme le travail sur la langue effectué par l’auteur.

D’autres traducteurs défendent la traduisibilité de ces langues et proposent de les remplacer par d'autres existantes dans le territoire de la langue-cible. C'est le cas, par exemple, de Julià (Hurtado Albir, 2017), qui soutient que, de la même manière que le lecteur d'un roman traduit est conscient que les personnages ne devraient pas parler sa langue, celui-ci doit aussi comprendre que, même si le traducteur utilise une langue minoritaire pour traduire les éléments hétérolingues du texte, ce personnage ne s'exprime pas dans cette langue dans le texte-source.

Cependant, à notre avis, l'utilisation du créole dans $B C$ n'a pas comme fonction - au moins, comme fonction principale - de montrer l'inscription sociale ou géographique des personnages, comme c'est le cas dans le texte analysé par Julià. Au contraire, il a des implications plus profondes qui senracinent dans une revendication identitaire et du droit d'exister au-delà des forces centripètes occidentales. Ainsi, la traduction par d'usages locaux de la langue-cible serait une façon de naturaliser l'Autre, considéré comme dérangeant pour le lecteur-cible. Cette position relève de l'égocentrisme, qui " conduit à l'exaltation de Soi, la fermeture à l'Autre et au déni de ce dernier " (Bernabé 2016 : 78), autant d'attitudes qui vont à lencontre du modèle de traduction qui serait censé se développer à travers la mondialité.

Ces solutions nétant pas satisfaisantes, nous considérons utile de passer en revue les stratégies de traduction existantes pour les termes hétérolingues selon Myriam Suchet (2009), afin de cerner leur relevance dans la traduction de $B C$. Il sagit de : la glose, où lélément hétérolingue est suivi d'une virgule et d'une traduction en « langue standard »; la synonymie, où lélément hétérolingue est suivi d'un synonyme en « langue standard » ; la contextualisation, qui consiste à disséminer dans le contexte des éléments qui permettraient au lecteur de comprendre le terme; labsence de traduction.

D’abord, la glose ne nous semble pas pertinente dans le cas qui nous occupe. Certes, il existe des auteurs qui utilisent ce procédé dans leur texte, c'est-à-dire qui traduisent l'élément hétérolingue dans la langue principale utilisée. Étienne Dassi (2007) explique que, souvent, lécrivain accompagne l'ethnolexème ${ }^{5}$ d'une glose pour essayer de faire passer le plus des valeurs ethnolinguis- 
tiques possibles véhicules par ce mot. Cependant, il ajoute que ces procédés appartiennent plutôt au génie de la langue française et que, dans d'autres cas, les gloses se montrent inefficaces pour rendre une explication suffisante de l'ethnolexème. Ce n'est pas le cas dans $B C$, où les éléments hétérolingues apparaissent le plus fréquemment sans explications ni gloses. Ainsi, l'auteur introduit consciemment de l'opacité dans son texte et rend difficile sa compréhension pour un lecteur non créolophone.

De ce fait, ajouter des gloses dans la traduction nous semblerait une intrusion dans le texte et une manière d'effacer son opacité. En outre, se poserait la question des éléments qu'il faut expliquer car, comme le soulignent Ashcroft et al., " in one sense virtually everything that happens or everything that is said can be ethnographic » (1999: 61). De plus, selon Chantal Zabus (2018), ce procédé peut être perçu comme agaçant chez certains lecteurs, qui verraient leur lecture constamment interrompue par ces explications.

Concernant la synonymie, elle nous semble avoir les mêmes problèmes que la glose, d'autant plus qu'un synonyme ne recouvre jamais la totalité de la signification de l'autre terme. Ainsi, ce procédé alourdirait la lecture sans s'avérer véritablement efficace dans son objectif.

La contextualisation est un procédé qui répond, d’après Dassi (2007), au légitime besoin ressenti par lécrivain de greffer des cours de civilisation et d'ethnolinguistique à son roman, afin de le rendre compréhensible pour des lecteurs qui ne font pas partie de sa même réalité. Mais ceci suppose encore un ancrage au centre, un écrire en fixant les yeux sur la métropole, en attendant son approbation. C’est une technique qui prime le confort du lecteur «métropolitain » et qui rend les éléments hétérolingues étrangers dans son propre univers culturel (Zabus 2018). En outre, cette technique peut amener l'auteur à faire des détours dans la trame du récit afin de contextualiser les éléments qui lui semblent de difficile compréhension. En conséquence, étant donné qu'Alfred Alexandre n'utilise pas systématiquement de contextualisation dans son roman, il nous semblerait une erreur et une soumission aux centres dominants de l'employer aux endroits opaques du texte.

Il nous reste à analyser la pertinence de la dernière des stratégies énoncée par Suchet : l'utilisation de mots non traduits dans le récit. Aux premiers abords, cette proposition peut sembler contraire à l'objectif même de la traduction : rendre un texte-source dans une langue-cible. En effet, comme le signale Venuti (1995), une traduction est souvent jugée comme acceptable quand elle peut se lire aisément, quand elle semble un texte transparent, sans péculiarités stylistiques ou linguistiques. Cependant, notre intention en traduisant $B C$ n'est pas de créer un texte qui pourrait avoir été écrit dans n'importe quelle autre culture, mais de montrer que le texte est bel et bien une traduction, afin de renvoyer le lecteur à la culture-source.

Une bonne manière d'y parvenir, nous semble-t-il, est d'insérer dans le texte traduit des mots créoles, qui ont la capacité déveiller un sentiment de surprise chez de lecteur-cible et de le renvoyer à un autre univers culturel, lui rappelant que l'œuvre n’appartient pas à sa culture. Ce type de traduction essaye de restreindre la violence ethnocentrique (Venuti 1995) toujours présente en traduction et de susciter chez le lecteur l'envie de se rapprocher de la culture-source pour mieux comprendre le récit. Ainsi, en complexifiant l'accès immédiat à la signification des mots créoles, le lecteur du texte traduit devra faire un effort de découverte de la culture-source. Cette technique est aussi une sorte de rébellion linguistique qui donne à voir les marges et qui se refuse à les noyer dans une traduction neutre. Elle permet en outre de rendre visible la situation actuelle du monde, où les cultures et les langues, loin de rester enfermées dans un seul pays, cohabitent et se côtoient 
au quotidien. Avec Glissant, nous nous demandons : «le monde, dans son unité éclatée, ne requiert-il pas que chacun s'efforce vers l'opacité reconnue de l'autre ?» (1981:18). Et nous avons envie de répondre : « oui ».

Nous proposons aussi, afin de marquer encore plus l'appartenance de ces mots à une autre langue, d'y introduire un balisage, même si celui-ci napparaît pas dans $B C$, en les écrivant en italique. Ceci contribuerait à attirer l'attention du lecteur-cible sur ces mots qui véhiculent une identité autre et sur le langage lui-même.

En outre, concernant les créations lexicales, nous considérons que le traducteur doit aussi mettre en ouvre des stratégies de création, tout comme l’auteur du roman, afin de greffer dans le texte-cible des éléments de la langue-source. Ces stratégies peuvent consister à la création de mots avec une base créole ou à l'imitation dans la langue-cible d'expressions calquées sur celles du français des Antilles, par exemple, selon le procédé employé par l'auteur lui-même ${ }^{6}$. En outre, ceci contribue aussi à modifier la langue majeure. Labsence de traduction de certains mots permet alors d'identifier l'appartenance du texte à une culture autre.

\section{Conclusion}

La mondialité a changé la façon dont des populations se mettent en relation, permettant une plus grande ouverture envers l'Autre et transformant les modèles de traduction eurocentrés qui régnaient jusqualors en Europe. Les auteurs postcoloniaux, dont Alfred Alexandre, se servent des stratégies hétérolingues afin d’affirmer leur identité plurielle.

Ainsi, pour traduire $B C$, il est fondamental de comprendre l'utilité de ces stratégies dans la revendication identitaire et d’agir en conséquence, en évitant de hiérarchiser les langues et de donner à la langue majeure une position privilégiée par rapport à la langue mineure, car toutes les deux ont dans le récit une position d'égalité.

Nous proposons que, puisque l'une des caractéristiques fondamentales de la mondialité est le contact fertile entre les langues et les cultures, le texte-cible doit être aussi hétérolingue, pour refléter la culture-source dans laquelle deux langues cohabitent. Ainsi, il nous semble intéressant de ne pas traduire les mots créoles présents dans le texte, tout en les mettant en valeur à travers un balisage. Ainsi, le lecteur-cible pourra être conscient de l'existence d'une autre langue dans le texte et comprendre qu'il s'agit d'une traduction d'une culture différente de la sienne, même s'il peut lire le récit dans sa langue. En outre, cette stratégie permet de conserver l'opacité du texte, introduite volontairement par l'auteur, car il n'explique pas systématiquement la signification des mots créoles dans son récit.

Certes, le lecteur-cible expérimentera toujours une sorte de dépaysement, une certaine étrangeté face à la nouvelle réalité qu'il découvrira dans le texte. Mais c'est cette sensation même que nous éprouvons au contact avec d'autres cultures et qui était si chère à Victor Segalen (1978). De plus, nous pensons que montrer une culture de la façon la plus proche possible de sa réalité est susceptible déveiller de la curiosité chez le lecteur, qui pourrait être tenté de comprendre et de se rapprocher de cette culture qu'il vient de découvrir.

6 Il nous semble que, pour une éventuelle traduction de Bord de Canal dans une langue autre que l'espagnol d'Espagne, la typologie des problèmes serait identique et répondrait à la même grille d’analyse. 


\section{Références bibliographiques}

Affergan, F. (2006). Martinique : les identités remarquables. Anthropologie d’un terrain revisité. Paris : Presses universitaires de France.

Alexandre, A. (2004). Bord de Canal. Paris : Dapper.

. (2010). Les villes assassines. Paris : Écritures.

Anderson, J. (2017). A Kumara by any other name: literary translation in and of the Polynesian Pacific. In S. Bertaco (Éd.), Translating the Postcolonial in Multilingual Contexts (pp. 21-38). Montpelier : Presses universitaires de la Méditerranée.

Ashcroft, B.; Griffiths, G.; \& Tiffin, H. (1989). The Empire Writes Back. Theory and practice in post-colonial literatures. London/New York: Routledge.

Berman, A. (1992). L'Epreuve de l'étranger. Culture et traduction dans l'Allemagne romantique. Paris : Gallimard.

Bernabé, J. (2016). La dérive identitariste. Paris : L’Harmattan.

Calvet, L.-J. (2001). Linguistique et colonialisme. Paris : Payot et Rivages.

Chamoiseau, P. (1994). Chemin-d’école. Paris : Le grand livre du mois. . (1997). Écrire en pays dominé. Paris : Gallimard.

Confiant, R. (2007). Dictionnaire créole martiniquais-français. Guyane : Ibis rouge.

. (2016). Traduire la langue dominée en contexte de mondialisation. In G. L'Étang, \& C. Mencé-Caster (Éds.), Écrire la domination (pp. 231-255). Paris : Caraïbéditions-Université.

Dassi, É. (2007). Des gloses interlinéaires socioculturalisées à la question de lécriture romanesque africaine francophone. Sudlangues, 6, 89-106. <http://www.sudlangues.sn, consulté le 11/03/2020>.

Deleuze, G. ; \& Guattari, F. (1975). Kafka. Pour une littérature mineure. Paris : Éditions de minuit. . (1980). Mille plateaux. Paris : Éditions de minuit.

Glissant, É. (1975). Malemort. Paris : Éditions du Seuil.

- (1997 [1981]). Le discours antillais. Paris : Gallimard.

. (2005). La cohée du Lamentin. Poétique IV. Paris : Gallimard.

Grutman, R. (1997). Des langues qui résonnent. L’hétérolinguisme au XIXe siècle québécois. Québec: Fides.

Hurtado Albir, A. (2001). Traducción y traductología. Introducción a la Traductología. Madrid : Cátedra.

Mambi Magnack, J. M. (2013). Littérature postcoloniale et esthétique de la folie et de la violence: une lecture de neuf romans africains francophones et anglophones de la période post-indépendance. Thèse doctorale, SaintÉtienne, Université Jean Monnet. <https://tel.archives-ouvertes.fr/tel-01063597>.

Nida, E. (1945). Linguistics and Ethnology in Translation-Problems. WORD, 1, 2, 194-208, doi : 10.1080/00437956.1945.11659254.

Oustinoff, M. (2011). Traduction et mondialisation. Paris : CNRS Éditions. <doi:10.4000/books.editionscnrs.15039>.

Robinson, D. (1997). Translation and Empire. Postcolonial Theories Explained. Manchester: St. Jerome.

Segalen, V. (1955). Essai sur l'exotisme. Paris : Le livre de poche.

Suchet, M. (2009). Outils pour une traduction postcoloniale. Littératures hétérolingues. Paris : Édition des archives contemporaines.

Venuti, L. (1995). The Translator's Invisibility. Londres : Routledge. 
Véronique, G. D. (2000). Créole, créoles français et théories de la créolisation. L'Information Grammaticale, 85, 33-38. <doi:10.3406/igram.2000.2769>.

Zabus, C. (2018). Le palimpseste africain. Indigénisation de la langue dans le roman ouest-africain europhone. M. Labbé, \& R. Théri (Trad.). Paris : Karthala. 